\title{
Circ_0026344 overexpression inhibits the migration, invasion, and enhances apoptosis of colorectal cancer cells by sponging miR-31
}

\section{Ye Jin}

Zhejiang Litongde Hospital

Lingli Yu

Affiliated Children's Hospital of Zhejiang University Medical College

\section{Bin Zhang}

Zhejiang Litongde Hospital

\section{Yun Chen}

Zhejiang Litongde Hospital

Changfeng Liu

Zhejiang Litongde Hospital

\section{Zhi Chen}

Zhejiang Litongde Hospital

\section{Xiaobo Zhan}

Zhejiang Litongde Hospital

Qi Wu ( $\nabla$ wuqi_qiwqiwi@163.com )

Zhejiang Litongde Hospital

\section{Research}

Keywords: Colorectal cancer, Circ_0026344, MiR-31, Apoptosis, Migration, Invasion

Posted Date: August 17th, 2020

DOI: https://doi.org/10.21203/rs.3.rs-58633/v1

License: (c) (1) This work is licensed under a Creative Commons Attribution 4.0 International License. Read Full License 


\section{Abstract}

Background: Circ_0026344 was reported to be associated with the metastasis of colorectal cancer (CRC). This study aimed to investigate the expression of circ_0026344 in CRC and the effect mechanisms of circ_0026344 on CRC.

Methods: The expressions of circ_0026344 and miR-31 in clinical CRC tissues or CRC cell lines were analyzed by qPCR. The target of circ_0026344 was predicted and verified by Circlnteractome and dualluciferase reporter assays. The correlation between circ_0026344 and miR-31 expression was analyzed using Pearson analysis. After the CRC cells were overexpressed circ_0026344 or miR-31 or silenced circ_0026344, the viability, apoptosis, migration, and invasion of CRC cells were evaluated by CCK-8, flow cytometry, wound healing, and transwell. Also, the expressions of miR-31, Bcl-2, Bax, E-cadherin, and Ncadherin in the cells were detected by qPCR or Western blot.

Results: Circ_0026344 was low-expressed in CRC tissues and cell lines. Circ_0026344 sponged miR-31 which was high-expressed in CRC tissues. The expression of circ_0026344 was negatively correlated to the expression of miR-31. The miR-31 expression could be down-regulated by circ_0026344 overexpression. Circ_0026344 overexpression inhibited the cell viability, migration, and invasion; and enhanced the apoptosis of CRC cells. Circ_0026344 overexpression decreased the expressions of Bcl-2 and $\mathrm{N}$-cadherin and increased the expressions of Bax and E-cadherin in CRC cells. Circ_0026344 silencing and miR-31 overexpression had an opposite effect on CRC cells as circ_0026344 overexpression. Furthermore, miR-31 overexpression counteracted the effect of circ_0026344 overexpression.

Conclusion: Circ_0026344 overexpression inhibited the migration, invasion, and enhanced apoptosis of CRC cells by sponging miR-31.

\section{Background}

Colorectal cancer (CRC), including rectal cancer and colon cancer, is one of the common malignant tumors of the digestive tract in the clinic (1). Symptoms are not obvious in the early stage, but in the late stage, symptoms such as blood in the stool, diarrhea, local pain anemia, weight loss, and cachexia will appear, which seriously affects the quality of life of the patient (2). Although modern medicine has expanded the scope of surgical operations and improved surgical methods, the overall survival rate of CRC has not improved $(1,3)$. Therefore, the novel approach is urgently needed to be developed to improve the current status of CRC treatment.

Circular RNA (circRNA), a kind of non-coding RNAs, which is featured by a covalently closed-loop structure and does not hold the $5^{\prime}$ and $3^{\prime}$ ends contained in linear RNAs (4). In the recent decade, a number of evidence reported that circRNA owns a crucial function in the development of all kinds of cancers, including CRC cancer $(4,5)$. For instance, circ-ANF609 enhances the migration of CRC cells by down-regulating Gli1 (6), circ_0014717 was down-regulated in CRC and its overexpression suppresses the tumor growth of CRC through up-regulating p16 (7), and circ_0009361 inhibited the progression of CRC 
by regulating APC2 (8). Circ_0026344, a newly discovered circRNA, was previously proved to be lowexpressed in gastric cancer and it can be an independent predictor for CRC patients' overall survival (9). What's more, circ_0026344 was suggested that its abnormal expression was associated with the metastasis of CRC (10), although the expression level and effect of circ_0026344 on CRC needed more exploration.

Scholars have proved that circRNA is able to function as a sponge of miRNA and thereby to regulate the progression of different kinds of diseases and cancers, including CRC (11-13). In particular, circ-ITGA7 inhibits the proliferation of CRC cells by sponging miR-3187-3p (14), circ_0009361 acts as a sponge of miR-582 to inhibit the processes of CRC (8), circ_0000523 modulates the proliferation and apoptosis of CRC cells through acting as a sponge of miR-31 (13). MiR-31, highly expressed in CRC, is widely proved that it is correlated to the metastasis of CRC and has a high value in CRC diagnosis $(15,16)$. Whether circ_0026344 had an effect on CRC by sponging miR-31 needs further exploration and verification.

Based on the present background, in this study, we tried to uncover the specific role of circ_0026344 on CRC and further to verify the potential relationship that circ_0026344 could sponge miR-31 in CRC.

\section{Methods}

\section{Clinical tissues and ethics statement}

Cancer tissues and corresponding adjacent tissues were obtained from patients with CRC $(n=40)$ who have received surgery from Zhejiang Litongde Hospital. The use of the tissues was approached by the Ethics Committee of Zhejiang Litongde Hospital (Z20200121X) and informed consent was signed by all patients.

\section{Cell culture}

Normal human colorectal mucosal cell line FHC (CRL-1831) and human CRC cell lines including HCT-15 (CCL-225), SW480 (CCL-228), SW620 (CCL-227), and HCT116 (CCL-247) were obtained from American Type Culture Collection (ATCC; Manassas, Virginia, USA). FHC cells were cultured in DMEM:F12 medium (30-2006, ATCC, Manassas, Virginia, USA) containing 10\% fetal bovine serum (FBS; 30-2020, ATCC, Manassas, Virginia, USA) and $20 \mathrm{ng} / \mathrm{mL}$ human recombinant EGF (PHG0311, Gibco, Waltham, Massachusetts, USA). HCT-15 and HCT116 cells were cultured in RPMI 1640 medium (30-2001, ATCC, Manassas, Virginia, USA) containing 10\% FBS. SW480 and SW620 cells were cultured in Leibovitz's L-15 medium (30-2008, ATCC, Manassas, Virginia, USA) containing 10\% FBS. All cells were incubated in a $37^{\circ} \mathrm{C}$ atmosphere with $5 \% \mathrm{CO}_{2}$.

\section{Transfection}

Small interfering RNA for circ_0026344 (si-circ_0026344; 5'-GAGAGGACUCAGGAUUUAAC-3'), plasmids overexpressing circ_0026344 (junction site: 5'-GTTAAATCCTGAGTCCTCTC-3') ligated into pLV-cir vector were synthesized from Miaoling Biological (Wuhan, China, http://www.miaolingbio.com/). Vector of pLV- 
cir (Vector) without any target sequence was used as a negative control for circ_0026344 silencing and overexpression. MiR-31 mimic (5'-UCGAUACGGUCGUAGAACGGA-3') and mimic control (5'UUCUCCGAACGUGUCACGUUU-3') were brought from GenePharma (Shanghai, China). Before transfection, the $1.0 \times 10^{6} \mathrm{SW} 480$ and HCT116 cells in $2 \mathrm{~mL}$ medium were plated into per well of a 6-well plate, respectively. After the cell confluence arrived about $80 \%$, the $2 \mu \mathrm{g}$ si-circ_0026344, plasmids, or miR31 mimic were transfected into the cells by using Lipofectamine 3000 Transfection Reagent (L3000015, Invitrogen, Waltham, Massachusetts, USA) in light of the instruments. Finally, the cells were harvested for later use after being transfected for $48 \mathrm{~h}$.

\section{Dual-luciferase reporter assay}

Based on the prediction of Circular RNA interactome (Circlnteractome; https://circinteractome.nia.nih.gov/index.html), binding sites existed between circ_0026344 with miR-31. The wide-type sequence of circ_0026344 (circ_0026344-WT) which contains binding sites of miR-31 (5'AGGTTCAAATCATTCTCTTGCCT-3') and the mutant sequence of circ_0026344 (circ_0026344-MUT; 5'AGGTTCAAATCATTCTACTGCTT-3') were inserted into the pGL3-basic vectors (VT1554, YouBio, Hunan, China) respectively.

Then, the $2.5 \times 10^{5}$ SW480 and HCT116 cells in $300 \mu \mathrm{L}$ medium were placed into per well of a 48-well plate, respectively. After the cell confluence arrived at about $80 \%$, the cells were transfected with miR-31 mimic and circ_0026344-WT or circ_0026344-MUT together. After transfection finished, the cells were collected and proceed with the dual-luciferase-reporter-kit (ab228530, Abcam, Cambridge, UK). Finally, the proceeded cells were placed into a SpectraMax reader (Molecular Devices, Shanghai, China) to measure the activity of the luciferase.

\section{CCK-8 assay}

After transfection, $1.0 \times 10^{4} \mathrm{SW} 480$ and HCT116 in $100 \mu \mathrm{L}$ medium were placed into a 96-well plate respectively and further cultured for $24 \mathrm{~h}, 48 \mathrm{~h}$, or $72 \mathrm{~h}$. Then, $10 \mu \mathrm{L}$ of CCK-8 buffer (A56097, OKA, Beijing, China) was added into each well of the 96-well plate to further culture the cells for $4 \mathrm{~h}$. Finally, the absorbance of per well of the 96-well plate was measured by a microplate reader (Imark; Bio-Rad, Hercules, California, USA) under $570 \mathrm{~nm}$.

\section{Flow cytometry}

Cell apoptosis of SW480 and HCT116 cells after transfection was detected using Annexin VFITC/Propidium lodide (PI) Kit (BB-4101-2, BestBio, Nanjing, China) by flow cytometry. In brief, after being 
transfected, SW480 and HCT116 cells were harvested and washed with PBS (PB180327, Procell, Wuhan, China) for three times. Then the cells were suspended in $500 \mu \mathrm{L}$ Working Buffer and stained with $5 \mu \mathrm{L}$ of Annexin V-FITC solution and $5 \mu \mathrm{L}$ of PI solution for $25 \mathrm{~min}$ in the dark. Finally, the cell apoptosis was analyzed by a FACSCaliburTM flow cytometer (BD Biosciences, Franklin Lake, New Jersey, USA).

\section{Wound healing assay}

After transfection, $2.0 \times 10^{6} \mathrm{SW} 480$ and HCT116 in $2 \mathrm{~mL}$ medium were placed into a 6-well plate respectively and cultured until the cell confluence arrived over $95 \%$. Then a wound was created in the each well, and each wound had equal width. Meantime, the previous medium was refreshed with $2 \mathrm{~mL}$ medium without FBS. The images of the wounds at 0 and $24 \mathrm{~h}$ were observed under an optical microscope (BX53, Olympus, Tokyo, Japan) under $100 \cdot$ magnification. Finally, cell migration rate was quantified by Image $\mathrm{J}$ software (Version 1.8.0).

\section{Transwell assays}

After transfection, $1.0 \times 10^{5}$ SW480 and HCT116 cells in $200 \mu \mathrm{L}$ medium without FBS were respectively placed transwell chambers (3422, Corning life sciences, Corning, New York, USA) which pre-coated with matrigel (354234, Corning life sciences, Corning, New York, USA). Transwell chambers were then inserted into a 24-well plate, meantime, $700 \mu \mathrm{L}$ medium with $15 \%$ FBS was added into the 24 -well plate and incubated the cells for $24 \mathrm{~h}$. Then, the cells inside the chambers were wiped and the cells outside the chambers were fixed with $4 \%$ paraformaldehyde (M1598, MREDA, Beijing, China) which followed by staining with crystal violet (M042723, MREDA, Beijing, China) for 20 min. Then the stained cells were observed under an optical microscope (BX53, Olympus, Tokyo, Japan). Finally, cell invasion rate was quantified by Image $\mathrm{J}$ software (Version 1.8.0).

\section{RNA extraction and Quantitative Real-time PCR (qPCR)}

The total RNAs in the clinical tissues and cultured cell lines were extracted using TRIzol (15596, Invitrogen, Waltham, Massachusetts, USA) for analyzing the expressions of circ_0026344. MiRNAs in the clinical tissues and cultured cell lines were also extracted using miRNA isolation regent (DP501, TianGEN, Beijing, China) for the analyzing the expression of miR-31. Then the RNAs we extracted were reversetranscribed into cDNA by using DNA synthesis regent (AE301-02, TransGen, Shanghai, China). Subsequently, the $1 \mu \mathrm{L}$ of cDNA were mixed with $4 \mu \mathrm{L}$ primers and $5 \mu \mathrm{L}$ supermix (AQ601-01, TransGen, Shanghai, China). Finally, the amplification reaction of cDNA in the mixed solution was detected and documented under the Q6 system (Applied Biosystems, CA, USA) in the light of the instrument. The primers involved in this experiments were as follows: circ_0026344-F: 5'-CTTTTGACCACAAGCCAGCC-3', 
circ_0026344-R: 5'-CCAGAGGACGTGCAAACTCT-3'; miR-31-F: 5'-AGCTGTCGTATCCAGTGCAA-3', miR-31-R: 5'-GTCGTATCCAGTGCGTGTCG-3'; GAPDH-F: 5'-GGAGCGAGATCCCTCCAAAAT-3', GAPDH-R: 5'GGCTGTTGTCATACTTCTCATGG-3'; U6-F: 5'-CTCGCTTCGGCAGCACA-3', U6-R: 5'AACGCTTCACGAATTTGCGT-3'. GAPDH was used as an internal control for circ_0026344 and U6 was used as an internal control for miR-31.

\section{Western blot assays}

After transfection, SW480 and HCT116 cells were incubated with NP-40 (M052443, MREDA, Beijing, China) for $15 \mathrm{~min}$ at $4^{\circ} \mathrm{C}$ and centrifuged $(14000 \cdot \mathrm{g})$ for $30 \mathrm{~min}$ at $4^{\circ} \mathrm{C}$. Then the protein was collected and its concentration was determined using a BCA kit (M052460, MREDA, Beijing, China). After that, $25 \mu \mathrm{g}$ protein were added into the $12 \%$ of 10 well SDS-PAGE gels (C-0047, Bioss, Beijing, China) for electrophoresis under 100 volts for $120 \mathrm{~min}$. After electrophoresis, the protein was transferred to the surface of $0.22 \mu \mathrm{m}$ of a PVDF membrane (M058646, MREDA, Beijing, China) and blocked with $5 \%$ defatted milk for $2 \mathrm{~h}$. Then, the membranes were further incubated with primary antibodies at $4{ }^{\circ} \mathrm{C}$ for 18 h: Bcl-2 (1:3000, 26 kDa, ab59348, Abcam, Cambridge, UK), Bax (1:3000, 21 kDa, ab32503, Abcam, Cambridge, UK), E-cadherin (1:10000, 97 kDa, ab40772, Abcam, Cambridge, UK), N-cadherin (1:3000, $130 \mathrm{kDa}$, ab18203, Abcam, Cambridge, UK), and GAPDH (1:3000, $36 \mathrm{kDa}$, ab8245, Abcam, Cambridge, UK). The next day, the membranes were further incubated with rabbit IgG (1:10000, ab205718, Abcam, Cambridge, UK) or mouse IgG (1:10000, ab205719, Abcam, Cambridge, UK) for $2 \mathrm{~h}$ at room temperature.

Lastly, after $200 \mu \mathrm{l}$ of detection solution (P0019, Beyotime, Shanghai, China) was added to the membrane to incubate $2 \mathrm{~min}$, the image signal was examined under the Image Lab 3.0 (Bio-Rad, Hercules, California, USA).

\section{Statistical analysis}

One-way ANOVA was used for comparison among multiple groups, Two-way ANOVA was used for comparison of cell viability among multiple groups, and Dunnet's test was used as post-hoc tests. Correlation between miR-31 expression with circ_0026344 expression in clinical samples was analyzed using Pearson analysis. All statistical analyses were implemented using Graphpad 8.0 software, measurement data were described by Mean \pm SD, and $P<0.05$ was considered statistically significant.

\section{Results}

\section{Circ_0026344 was low-expressed in CRC tissues and cell lines}

CRC tissues and its corresponding adjacent normal tissues were firstly collected for the analysis of circ_0026344 expression level. As the data displayed in Fig. 1A, the expression of circ_0026344 in CRC tissues was significantly lower than that in normal tissues $(P<0.001)$. Similarly, the expression of circ_0026344 in CRC cell lines (HCT-15, SW480, SW620, HCT116) were also lower than that in human normal colorectal mucosal cell line FHC (Fig. 1B, P<0.01). Among these CRC cell lines, the expression of 
circ_0026344 in the SW480 was the highest and in the HCT116 was the lowest, hence, we chose the two cell lines for later use.

\section{Circ_0026344 regulated the cell viability, apoptosis, migration, invasion of SW480 and HCT116 cells}

Subsequently, circ_0026344 overexpression plasmids and si-circ_0026344 were used to overexpress and silence circ_0026344 expression in SW480 (Fig. 2A) and HCT116 (Fig. 2B) cells. After overexpression circ_0026344, the cell viabilities at 24 h, 48 h, and $72 \mathrm{~h}$ both in SW480 (Fig. 2C) and HCT116 (Fig. 2D) cells were inhibited $(P<0.05)$; while, the cell viabilities were increased by si-circ_0026344. On the contrary, the cell apoptosis of SW480 (Fig. 2E-F) and HCT116 (Fig. 2G-H) cells were induced by circ_0026344 overexpression $(P<0.001)$ while inhibited by si-circ_0026344 $(P<0.01)$. Besides, the abilities of the two cells to migrate (Fig. 3A-D) and invade (Fig. 3E-H) were also inhibited by circ_0026344 overexpression ( $P$ $<0.001)$ and enhanced by si-circ_0026344 $(P<0.01)$.

Circ_0026344 regulated the expressions of factors related to apoptosis and epithelial-mesenchymal transformation (EMT) in SW480 and HCT116 cells

To confirm the present discovery, we further detected the expressions of factors related to apoptosis and EMT (Fig. 4). After overexpression circ_0026344, the expressions of Bcl-2 and N-cadherin both in SW480 (Fig. 4A-B) and HCT116 (Fig. 4C-D) cells were down-regulated $(P<0.001)$, and the expressions of the Bax and E-cadherin were up-regulated $(P<0.001)$. On the contrary, the expressions of $\mathrm{Bcl}-2$ and $\mathrm{N}$-cadherin both in SW480 and HCT116 cells were up-regulated by si-circ_0026344 $(P<0.001)$ while the expressions of the Bax and E-cadherin were up-regulated by si-circ_0026344 $(P<0.001)$.

Circ_0026344 acted as sponge of miR-31

After the binding sites between circ_0026344 with miR-31 were predicted by Circlnteractome (Fig. 5A), we further performed dual-luciferase reporter assays to verify that circ_0026344 might a sponge of miR-31. As shown in Fig. 5B-C, the luciferase activities both in SW480 and HCT116 cells were decreased after transfected with circ_0026344-WT and miR-31 mimic together $(P<0.001)$, while, no difference were found in the luciferase activities of the two cell lines after transfected with circ_0026344-MUT and miR-31 mimic together, which indicated that circ_0026344 might act as a sponge of miR-31 in CRC.

\section{MiR-31 was high-expressed in CRC tissues and its expression was negative correlated to circ_0026344 expression both in CRC tissues and normal tissues}

Then we evaluated the expression of miR-31 in CRC tissues (Fig. 6A) and found that miR-31 expression was higher than that in the normal tissues $(P<0.001)$. Furthermore, the expression of miR-31 in normal tissues (Fig. 6B) and in CRC tissues (Fig. 6C) were both negative correlated to the expression of circ_0026344. These results made us wonder that the effect of circ_0026344 on CRC cells was mediated by sponging miR-31.

MiR-31 overexpression counteracted the effect of circ_0026344 on the cell viability, migration, invasion, and the expression of miR-31 in SW480 and HCT116 cells 
To further make the deep mechanism underlying the effect of circ_0026344 on CRC, the miR-31 mimic was used to overexpress the expression of miR-31 both in SW480 (Fig. 7A) and HCT116 (Fig. 7B) cells. Then the expressions of miR-31 in the two cells were evaluated (Fig. 7C-D), as the results revealed that the expression of miR-31 was down-regulated by circ_0026344 overexpression $(P<0.001)$ and upregulated by miR-31 mimic $(P<0.001)$ when compared with the Vector + MC group; while, after cooverexpressed with circ_0026344 and miR-31 (the circ_0026344 + M group), the decreased miR-31 which down-regulated by circ_0026344 overexpression was further decreased by miR-31 mimic as compared to the circ_0026344 + MC group $(P<0.001)$. Cell viability was illustrated in Fig. 7E-F, the cell viabilities were decreased by circ_0026344 overexpression $(P<0.05)$ and increased by miR-31 overexpression $(P<0.01)$ as compared to the Vector + MC groups, while after co-overexpressed circ_0026344 and miR-31, the effect of circ_0026344 overexpression on cell viabilities was offset by miR-31 overexpression $(P<0.01)$. Similarly, the cell migration (Fig. 8A-D) and invasion (Fig. 8E-H) were also analyzed. As depicted in Fig. 8, the abilities of cells to migrate and invade were decreased by circ_0026344 overexpression $(P<0.05)$ and increased by miR-31 overexpression $(P<0.001)$ as compared to the Vector + MC group, while after cooverexpressed circ_0026344 and miR-31, the effects of circ_0026344 overexpression on cell migration and invasion were offset by miR-31 overexpression $(P<0.001)$. All these discoveries suggested that the effects of circ_0026344 on cell viability, migration, and invasion of CRC cells might be mediated by sponging miR-31.

\section{MiR-31 overexpression counteracted the effect of circ_0026344 on the expressions of factors related to apoptosis and EMT in SW480 and HCT116 cells}

Lastly, we evaluated the expressions of factors related to cell apoptosis and EMT to further confirm our above discoveries (Fig. 9). The effects of circ_0026344 overexpression on these factors were the same as the results showed in Fig. 4. Meantime, miR-31 mimic up-regulated the expressions of Bcl-2 and Ncadherin $(P<0.005)$, and down-regulated the expressions of Bax and E-cadherin $(P<0.05)$ both in SW480 (Fig. 9A-B) and HCT116 (Fig. 9C-D) cells. Furthermore, after co-overexpressed circ_0026344 and miR-31, the effects of circ_0026344 overexpression on these factors were offset by miR-31 overexpression.

\section{Discussion}

The growing number of pieces of evidence have proved that circRNA with abnormal expression regulates the development of different types of diseases and cancers, including CRC $(4,17)$. In CRC, circ_00038646, circ-ZNF609, circ_0004585, and circ_102958 are high-expressed and further promote the development of CRC $(5,6,18,19)$; circ_0000523, circDDX7, circ_0014717, and circ-ITGA7 are low-expressed and the silencing of these circRNAs can enhance the malignant abilities of CRC cells $(7,13,14,17)$. In the present study, we also found that circ_0026344 was down-regulated both in CRC tissues and cell lines, which suggested that the process of CRC might be regulated by circ_0026344 although the effects and mechanisms were needed more exploration. 
The extremely high recurrence rate and mortality of CRC can be attributed to the strong migration and invasion abilities of cancer cells and less apoptosis in cancer cells (20). Therefore, the key to treatment $\mathrm{CRC}$ is to inhibit the migration and invasion and induce the apoptosis of cancer cells. The biological activities of CRC cells including migration, invasion, proliferation, and apoptosis also can be regulated by circRNAs such as circ-100876, circ_00038646, circ_0000523, circ-ZNF609, and so on $(5,6,13,21)$. In the present study, we discovered that circ_0026344 overexpression suppressed the migration and invasion, and induced the apoptosis of CRC cells, while, circ_0026344 silencing did the opposite. The cell apoptosis, migration, as well as invasion, are occurred after the expression changes of the factors which are related to these biological activities $(22,23)$. Apoptosis can be successfully carried out only after Bcl2 (one of the anti-apoptotic factors) binding with Bax (one of the pro-apoptotic factors) (22). During the intrinsic apoptosis of cells, the expression of Bcl-2 is decreased while Bax is up-regulated $(24,25)$. This study further proved that circ_0026344 overexpression down-regulated the expression of Bcl-2 and upregulated the expression of Bax in CRC cells, while, circ_0026344 silencing did the opposite. These phenomena further confirmed that circ_0026344 regulated the apoptosis of CRC cells. Equally important, EMT plays an important role in the process of cancer cells to migrate and invade (26). During this process, the cancer cells gradually lose the characteristics of epithelial cells with the expression decreasing of epithelial marker (E-cadherin), while, the cells gradually acquired the characteristics of mesenchymal cells with the expression increasing of mesenchymal markers ( $\mathrm{N}$-cadherin) (27). Thereby, the cancer cells can migrate and invade into the surrounding tissues and organs (28). Our study further discovered that circ_0026344 overexpression up-regulated the expression of E-cadherin and up-regulated the expression of $\mathrm{N}$-cadherin in CRC cells, while, circ_0026344 silencing did the opposite. These phenomena also confirmed that circ_0026344 did regulate the migration and invasion of CRC cells.

Since the role of "miRNA sponges" was put forward, circRNA was widely researched and proved that it can sponge some miRNAs to play its role in various biological activities (12). Theoretically, circ-ITGA7, circ_0009361, and circ_0000523 respectively sponge miR-3187-3p, miR-582, and miR-31 in CRC $(8,13$, 14). In the current study, we discovered that binding sites have existed between circ_0026344 with miR31, also, circ_0026344 down-regulated the expression of miR-31 in CRC cells, which indicated that the effect of circ_0026344 on CRC might be mediated by sponging miR-31. MiR-31 is previously found that it's highly expressed in CRC (15). Consistent with the report above, our results in this study also discovered that miR-31 was high-expressed in CRC tissues. What's more, miR-31 is proved to be correlated to the metastasis of CRC and can regulate autophagy, proliferation, and chemoresistance of CRC cells $(16,29,30)$. Similarly, in this study, the results confirmed that miR-31 overexpression not only promoted the migration, invasion, and suppressed the apoptosis of CRC cells but also counteracted the effect of circ_0026344. All the evidence revealed that circ_0026344 acted as a sponge of miR-31 to regulate the migration, invasion, and apoptosis of CRC cells.

\section{Conclusion}

To sum up, our study proved that circ_0026344 overexpression inhibited the migration and invasion, and induced apoptosis of CRC cells through sponging miR-31. Our findings in this study provided a novel 
approach for CRC diagnosis and treatment.

\section{Abbreviations}

$\mathrm{CRC}=$ colorectal cancer, circRNA $=$ Circular RNA

\section{Declarations}

\section{Competing interests}

The authors declare no conflicts of interest.

\section{Funding}

This work was supported by the [2020360772]; the Zhejiang Medical and Health Science and Technology Project

\section{Authors' contributions}

Substantial contributions to conception and design:

\section{Acknowledgements}

Not applicable.

\section{References}

1. Dekker E, Tanis PJ, Vleugels JLA, Kasi PM, Wallace MB. Colorectal cancer. Lancet. 2019;394(10207):1467-80.

2. The Lancet O. Colorectal cancer: a disease of the young? Lancet Oncol. 2017;18(4):413.

3. Zhong D, Li P, Gong PY. Hsa_circ_0005075 promotes the proliferation and invasion of colorectal cancer cells. Int J Biol Markers. 2019;34(3):284-91.

4. Liu J, Zhang X, Yan M, Li H. Emerging Role of Circular RNAs in Cancer. Front Oncol. 2020;10:663.

5. Du H, He Z, Feng F, Chen D, Zhang L, Bai J, et al. Hsa_circ_0038646 promotes cell proliferation and migration in colorectal cancer via miR-331-3p/GRIK3. Oncol Lett. 2020;20(1):266-74.

6. Wu L, Xia J, Yang J, Shi Y, Xia H, Xiang X, et al. Circ-ZNF609 promotes migration of colorectal cancer by inhibiting Gli1 expression via microRNA-150. J BUON. 2018;23(5):1343-9.

7. Wang F, Wang J, Cao X, Xu L, Chen L. Hsa_circ_0014717 is downregulated in colorectal cancer and inhibits tumor growth by promoting p16 expression. Biomedicine $\&$ pharmacotherapy $=$ Biomedecine \& pharmacotherapie. 2018;98:775 - 82.

8. Geng Y, Zheng X, Hu W, Wang Q, Xu Y, He W, et al. Hsa_circ_0009361 acts as the sponge of miR-582 to suppress colorectal cancer progression by regulating APC2 expression. Clinical science (London, 
England: 1979). 2019;133(10):1197 - 213.

9. Zhang X, Zhang L, Cui L, Chen M, Liu D, Tian J. Expression of circRNA circ_0026344 in gastric cancer and its clinical significance. Int J Clin Exp Pathol. 2020;13(5):1017-23.

10. Shen T, Cheng X, Liu X, Xia C, Zhang H, Pan D, et al. Circ_0026344 restrains metastasis of human colorectal cancer cells via miR-183. Artificial cells, nanomedicine, and biotechnology. 2019;47(1):4038-45.

11. Guan YJ, Ma JY, Song W. Identification of circRNA-miRNA-mRNA regulatory network in gastric cancer by analysis of microarray data. Cancer cell international. 2019;19:183.

12. Xiong DD, Dang YW, Lin P, Wen DY, He RQ, Luo DZ, et al. A circRNA-miRNA-mRNA network identification for exploring underlying pathogenesis and therapy strategy of hepatocellular carcinoma. Journal of translational medicine. 2018;16(1):220.

13. Jin Y, Yu LL, Zhang B, Liu CF, Chen Y. Circular RNA hsa_circ_0000523 regulates the proliferation and apoptosis of colorectal cancer cells as miRNA sponge. Brazilian journal of medical biological research $=$ Revista brasileira de pesquisas medicas e biologicas. 2018;51(12):e7811.

14. Yang G, Zhang T, Ye J, Yang J, Chen C, Cai S, et al. Circ-ITGA7 sponges miR-3187-3p to upregulate ASXL1, suppressing colorectal cancer proliferation. Cancer Manag Res. 2019;11:6499-509.

15. Cui Q. Significance of miR-27a and miR-31 in early diagnosis and prognosis of colorectal cancer. Oncol Lett. 2019;18(3):3092-6.

16. Zhang WW, Ming XL, Rong Y, Huang CQ, Weng $H$, Chen $H$, et al. Diagnostic Value Investigation and Bioinformatics Analysis of miR-31 in Patients with Lymph Node Metastasis of Colorectal Cancer. Anal Cell Pathol (Amst). 2019;2019:9740475.

17. Li XN, Wang ZJ, Ye CX, Zhao BC, Li ZL, Yang Y. RNA sequencing reveals the expression profiles of circRNA and indicates that circDDX17 acts as a tumor suppressor in colorectal cancer. Journal of experimental clinical cancer research: CR. 2018;37(1):325.

18. Tian J, Xi X, Wang J, Yu J, Huang Q, Ma R, et al. CircRNA hsa_circ_0004585 as a potential biomarker for colorectal cancer. Cancer Manag Res. 2019;11:5413-23.

19. Li R, Wu B, Xia J, Ye L, Yang X. Circular RNA hsa_circRNA_102958 promotes tumorigenesis of colorectal cancer via miR-585/CDC25B axis. Cancer Manag Res. 2019;11:6887-93.

20. Xu Y, Shen L, Li F, Yang J, Wan X, Ouyang M. microRNA-16-5p-containing exosomes derived from bone marrow-derived mesenchymal stem cells inhibit proliferation, migration, and invasion, while promoting apoptosis of colorectal cancer cells by downregulating ITGA2. Journal of cellular physiology. 2019;234(11):21380-94.

21. Zhang J, Wang H, Wu K, Zhan F, Zeng H. Dysregulated circRNA_100876 contributes to proliferation and metastasis of colorectal cancer by targeting microRNA-516b (miR-516b). Cancer Biol Ther. 2020;21(8):733-40.

22. Yang H, Liu JX, Shang HX, Lin S, Zhao JY, Lin JM. Qingjie Fuzheng granules inhibit colorectal cancer cell growth by the PI3K/AKT and ERK pathways. World J Gastrointest Oncol. 2019;11(5):377-92. 
23. Saitoh M. Involvement of partial EMT in cancer progression. J Biochem. 2018;164(4):257-64.

24. Houston JP. Apoptosis and autophagy. Cytometry A. 2019;95(6):655-6.

25. Voss AK, Strasser A. The essentials of developmental apoptosis. F1000Res. 2020;9.

26. Zhao GX, Xu YY, Weng SQ, Zhang S, Chen Y, Shen XZ, et al. CAPS1 promotes colorectal cancer metastasis via Snail mediated epithelial mesenchymal transformation. Oncogene. 2019;38(23):4574-89.

27. Derynck R, Weinberg RA. EMT and Cancer: More Than Meets the Eye. Developmental cell. 2019;49(3):313-6.

28. Brabletz T, Kalluri R, Nieto MA, Weinberg RA. EMT in cancer. Nature reviews Cancer. 2018;18(2):12834.

29. Yang $X, X u$ X, Zhu J, Zhang S, Wu Y, Wu Y, et al. miR-31 affects colorectal cancer cells by inhibiting autophagy in cancer-associated fibroblasts. Oncotarget. 2016;7(48):79617-28.

30. Du YL, Liang Y, Shi GQ, Cao Y, Qiu J, Yuan L, et al. LINC00689 participates in proliferation, chemoresistance and metastasis via miR-31-5p/YAP/ $\beta$-catenin axis in colorectal cancer. Experimental cell research. 2020:112176.

\section{Figures}
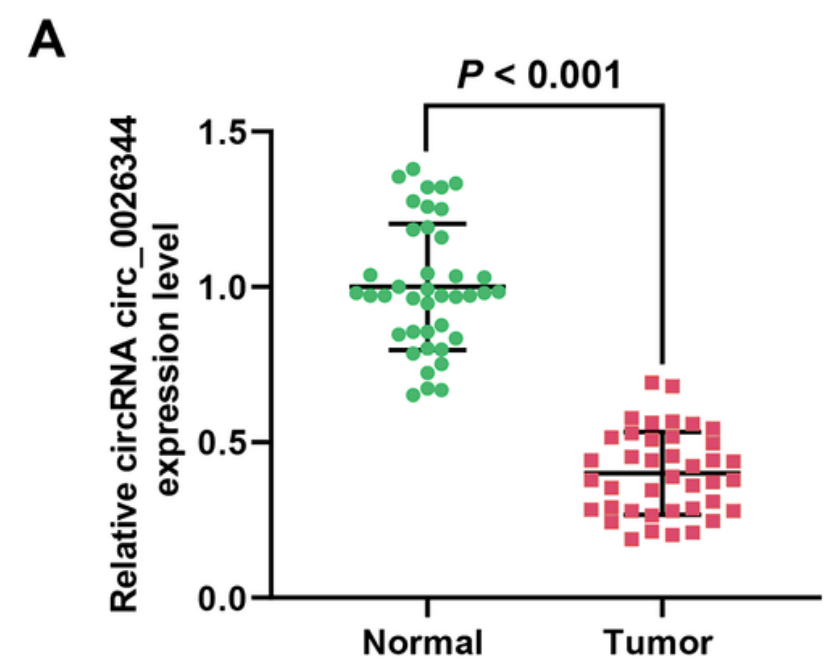

B

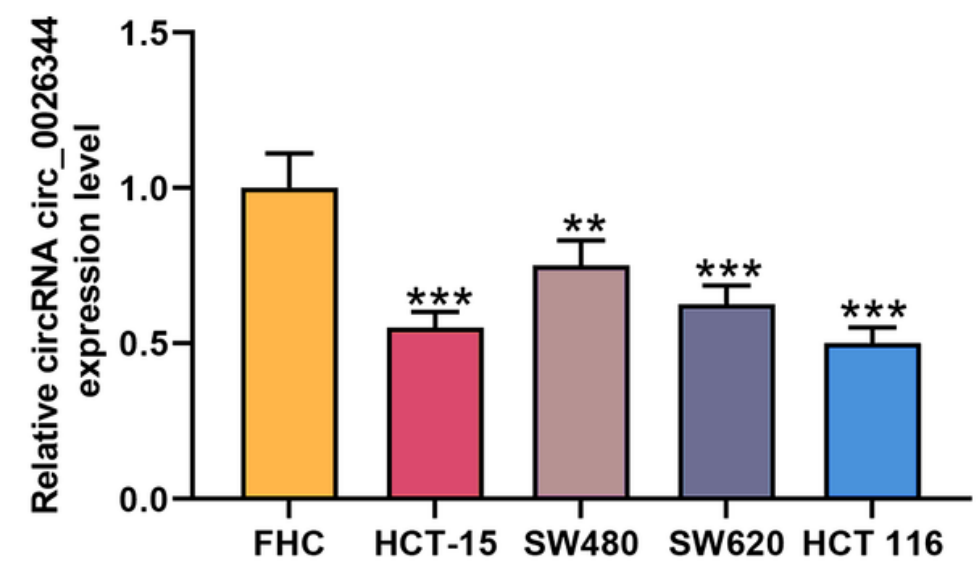

Figure 1

Circ_0026344 was low-expressed in CRC tissues and cell lines (A) The expression level of circ_0026344 in CRC tissues and adjacent normal tissues was detected by qPCR, GAPDH was used as an internal control. (B) The expressions level of circ_0026344 in CRC cell lines (HCT-15, SW480, SW620, HCT116) and human normal colorectal mucosal cell line (FHC) were detected by qPCR, GAPDH was used as an internal control. ( ${ }^{\star *} \mathrm{P}<0.01,{ }^{\star \star *} \mathrm{P}<0.001$, vs. FHC). (CRC: colorectal cancer, qPCR: Quantitative Real-time PCR) 


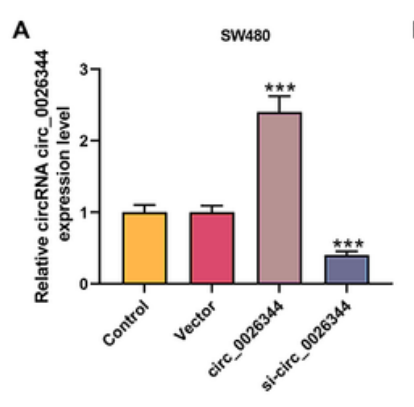

E

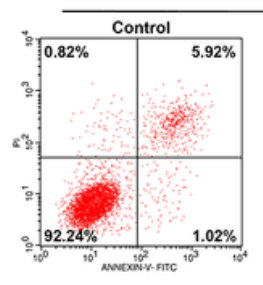

G

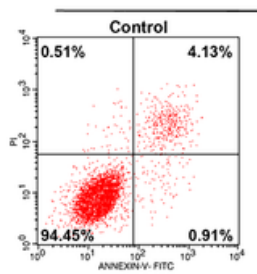

B

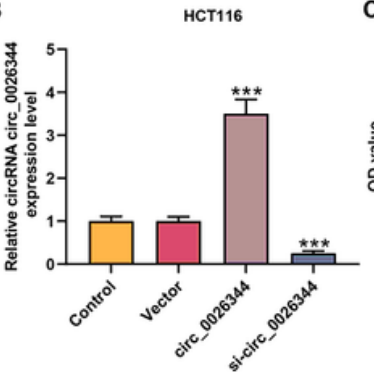

SW480

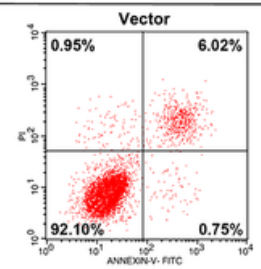

HCT116

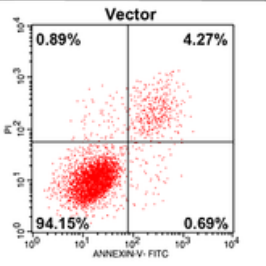

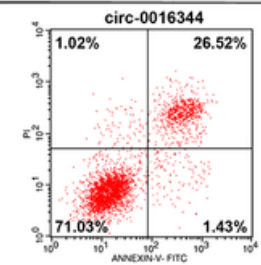

C
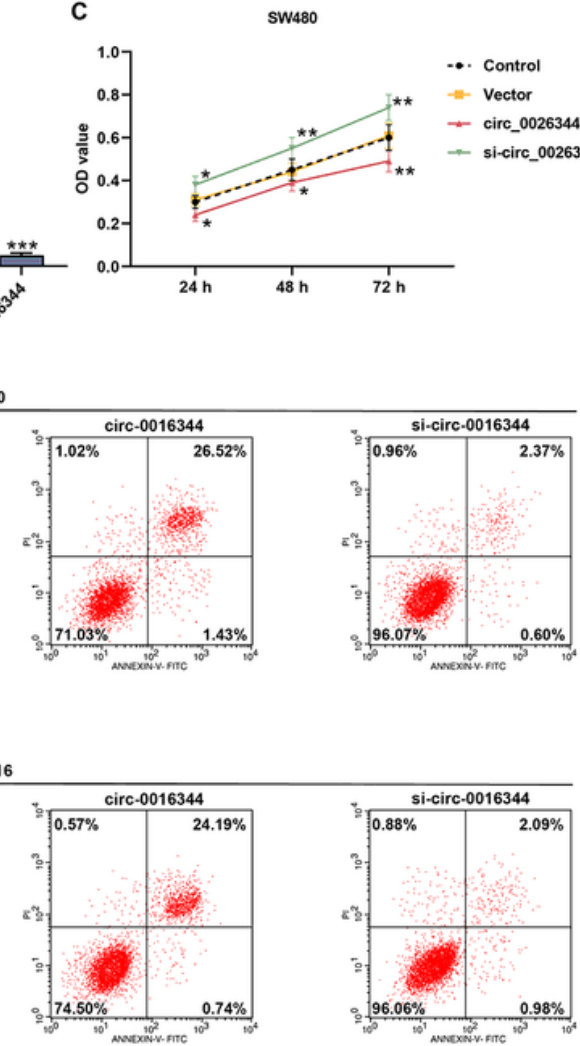

-. Control

- Vector

- circ_0026344

- si-circ_0026344
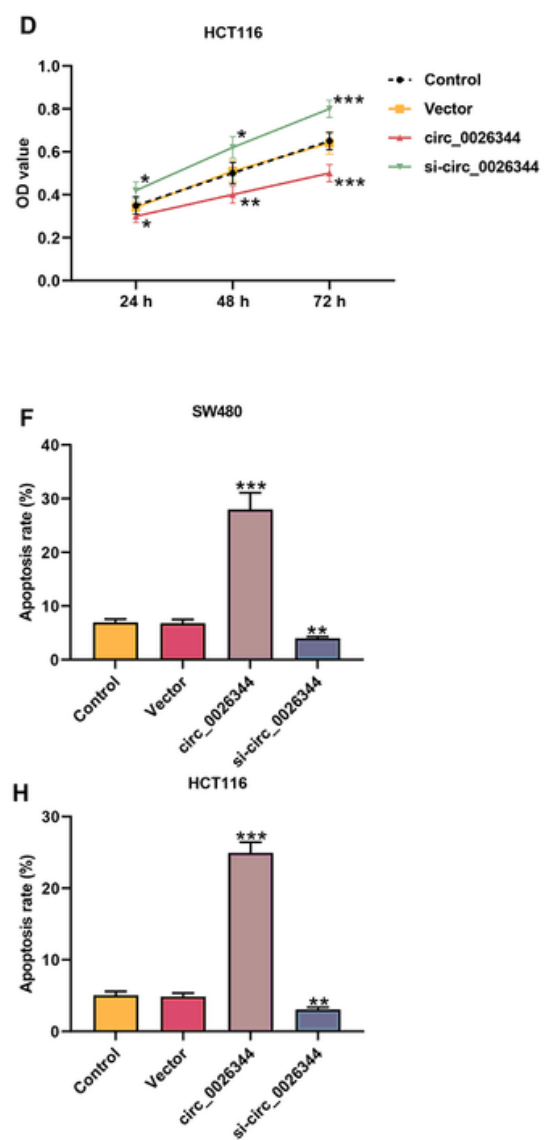

\section{Figure 2}

Circ_0026344 regulated the cell viability and apoptosis of SW480 and HCT116 cells (A-B) The transfection efficiency of circ_0026344 overexpression plasmids and si-circ_0026344 in SW480 (A) and HCT116 (B) cells were evaluated by qPCR, GAPDH was used as an internal control. (C-D) The viability of SW480 (C) and HCT116 (D) cells after being transfected and cultured for $24 \mathrm{~h}, 48 \mathrm{~h}$, and $72 \mathrm{~h}$ were detected by CCK-8 assays. (E-H) The apoptosis of SW480 (E-F) and HCT116 (G-H) cells after being

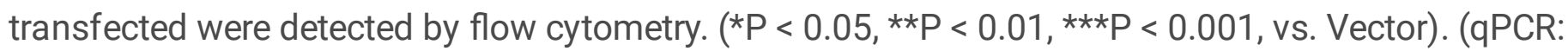
Quantitative Real-time PCR, si-circ_0026344: siRNA for circ_0026344) 


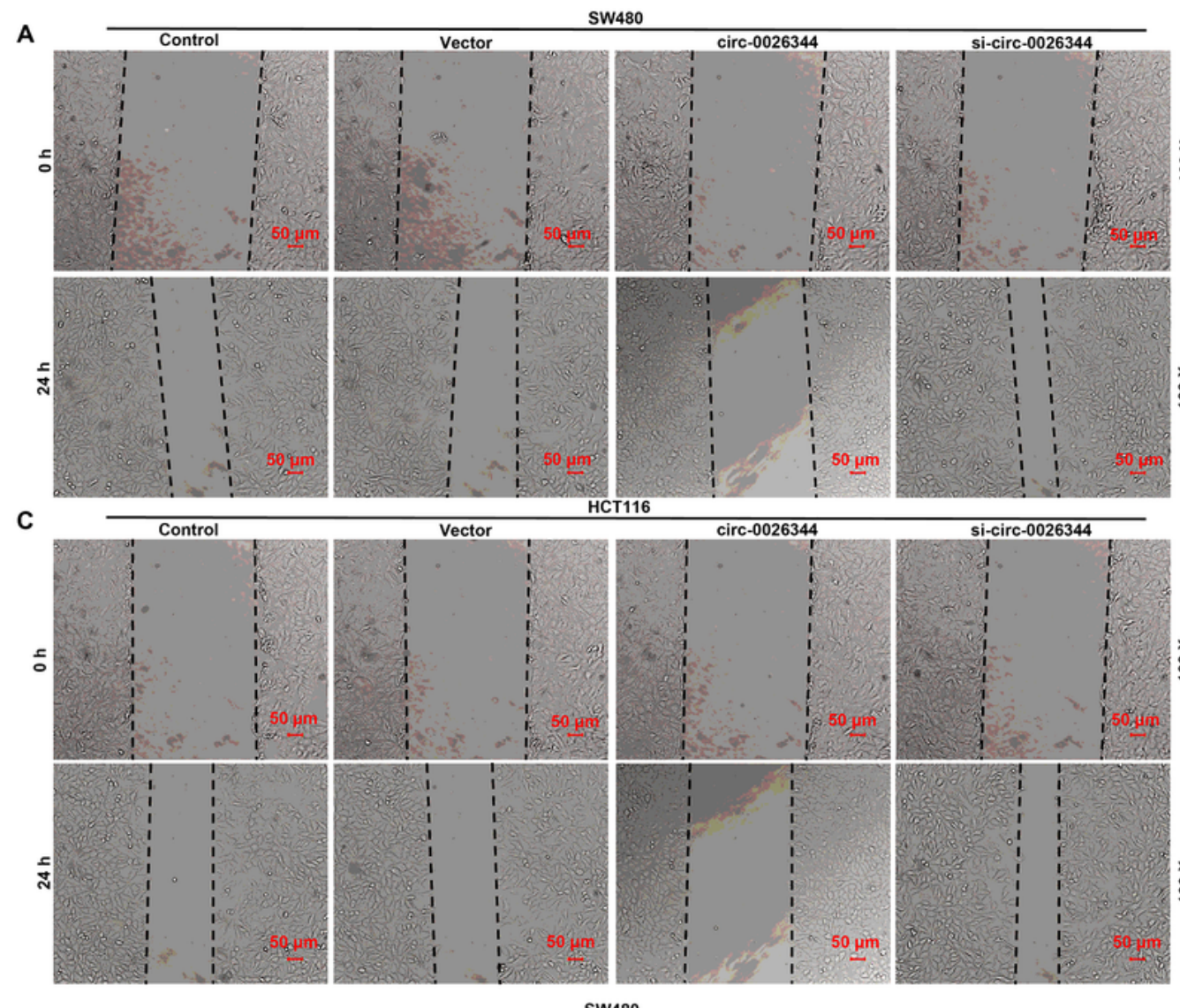

B
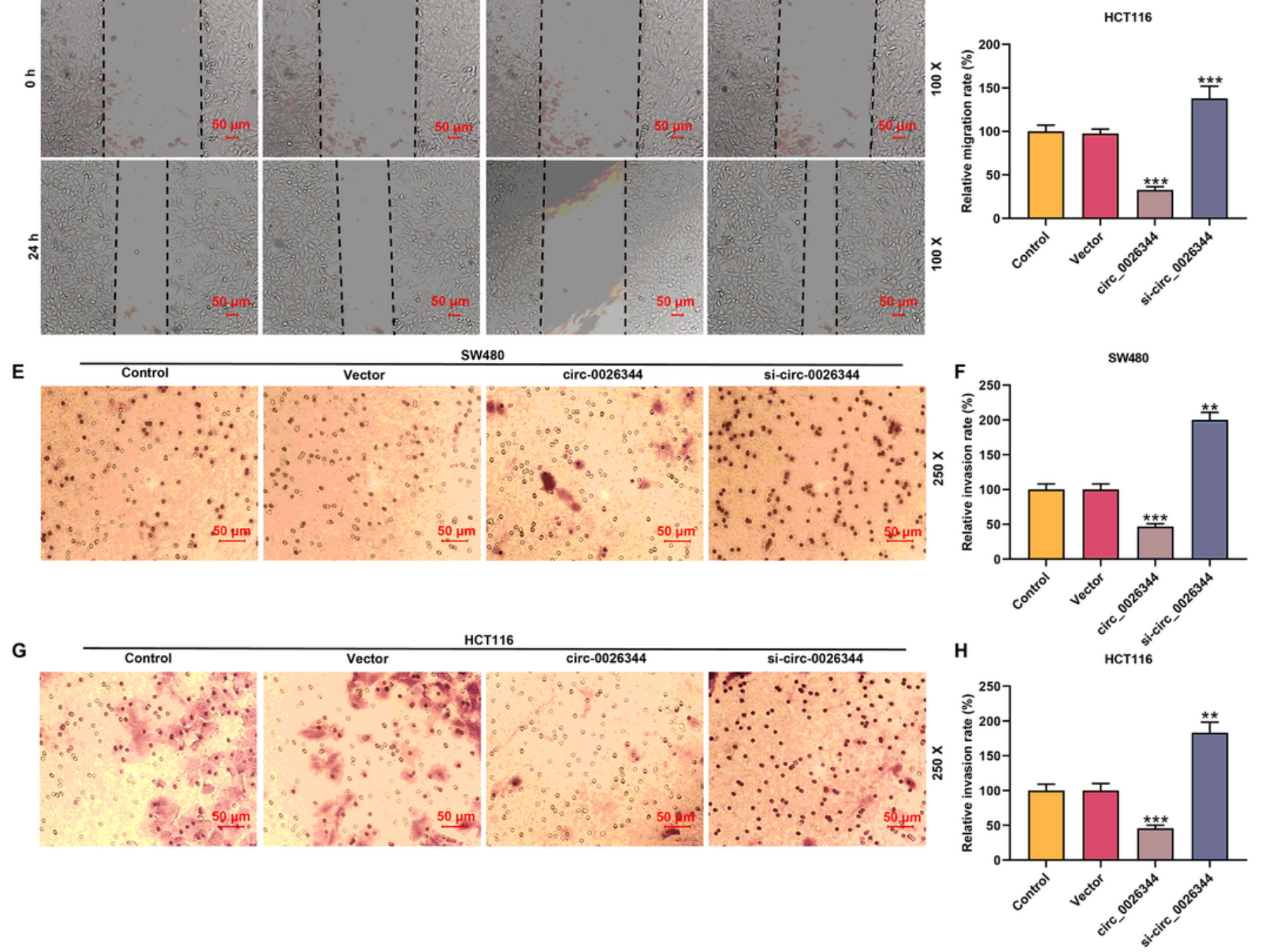

\section{Figure 3}

Circ_0026344 regulated the migration and invasion of SW480 and HCT116 cells (A-D) The migration of SW480 (C) and HCT116 (D) cells after being transfected were detected by wound healing assays. (E-H) The invasion of SW480 (E-F) and HCT116 (G-H) cells after being transfected were detected by transwell. $\left(\star \star P<0.01,{ }^{\star \star \star} P<0.001\right.$, vs. Vector). (si-circ_0026344: siRNA for circ_0026344) 

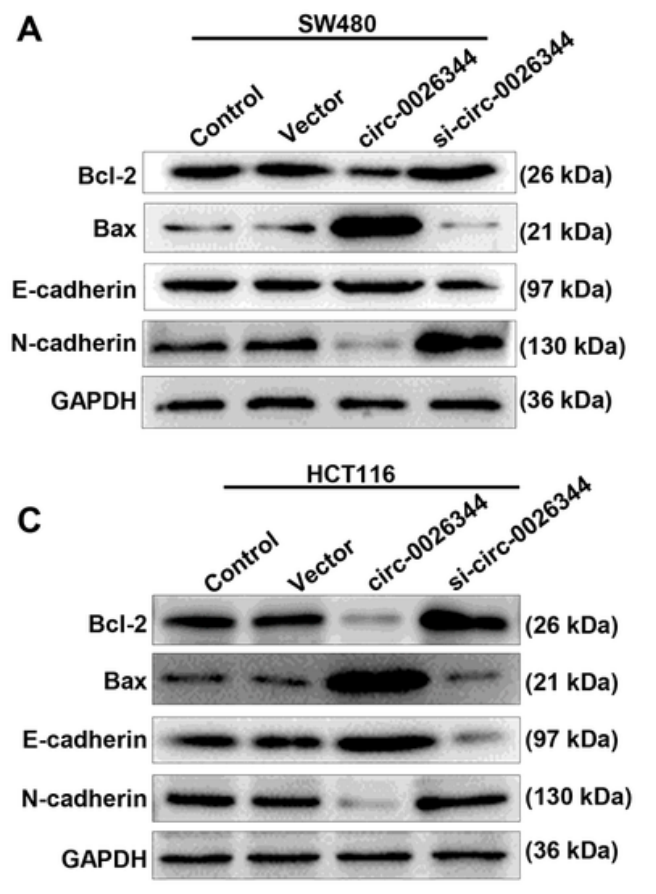

B

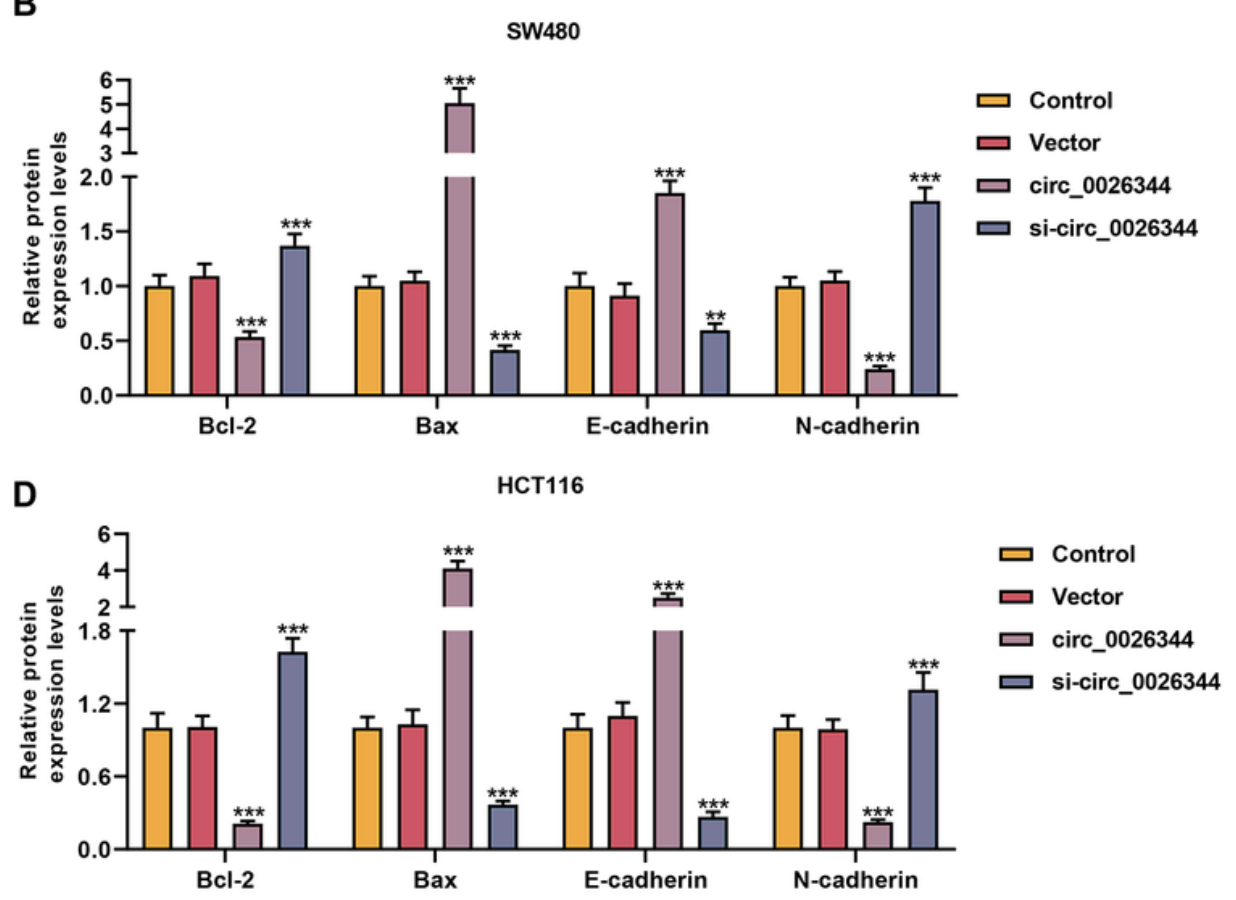

Figure 4

Circ_0026344 regulated the expressions of factors related to apoptosis and EMT in SW480 and HCT116 cells (A-B) The expressions of Bcl-2, Bax, E-cadherin, and N-cadherin in the SW480 cells after transfection were detected by Western blot, GAPDH was used as an internal control. (C-D) The expressions of Bcl-2, Bax, E-cadherin, and N-cadherin in the HCT116 cells after transfection were detected by Western blot, GAPDH was used as an internal control. (***P $<0.001$, vs. Vector). (si-circ_0026344: siRNA for circ_0026344, EMT: epithelial-mesenchymal transformation)

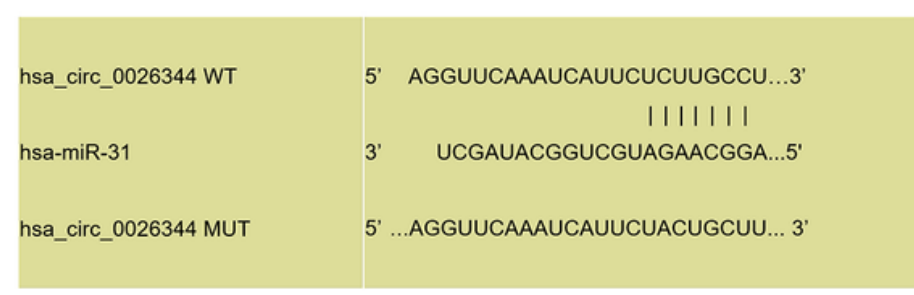

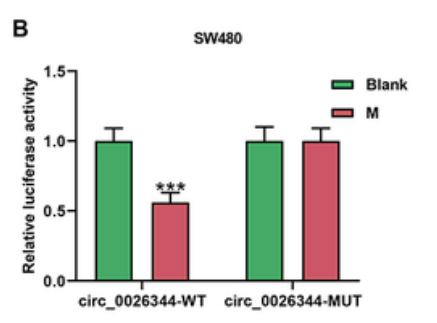

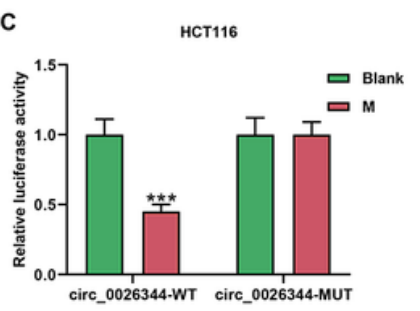

Figure 5

Circ_0026344 acted as sponge of miR-31 (A) The binding sites between miR-31 with circ_0026344 were predicted by Circlnteractome (https://circinteractome.nia.nih.gov/index.html). (B-C) Dual-luciferase reporter assays were performed to verify that miR-31 was a sponger of circ_0026344 both in SW480 (B) and HCT116 (C) cells. (***P $<0.001$, vs. Blank). (M: miR-31 mimic) 
A

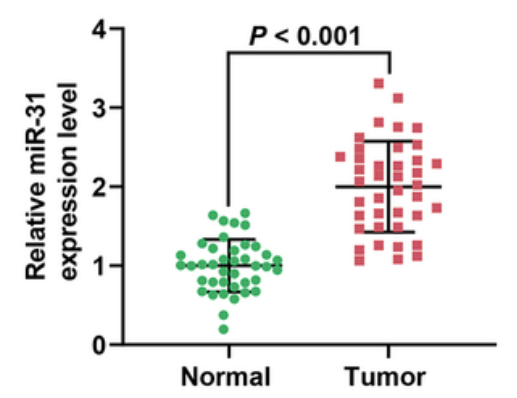

B

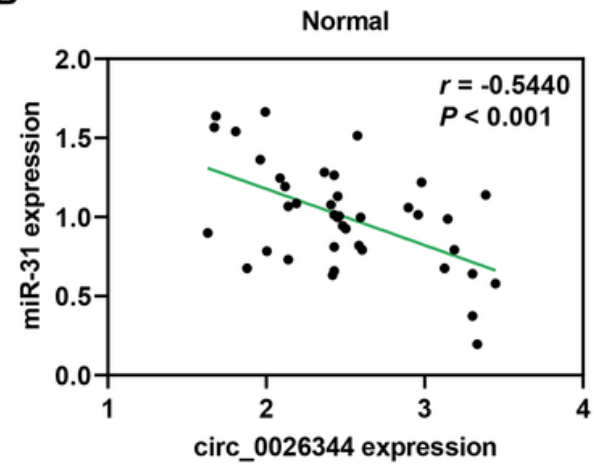

C

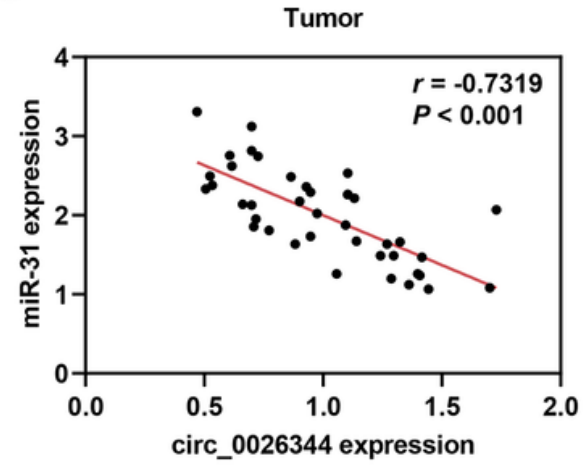

Figure 6

MiR-31 was high-expressed in CRC tissues and its expression was negative correlated to circ_0026344 expression both in CRC tissues and normal tissues (A) The expression level of miR-31 in CRC tissues and adjacent normal tissues was detected by qPCR, U6 was used as an internal control. (B-C) The correlation between miR-31 expression with circ_0026344 expression in normal tissues (B) and CRC tissues (C) were analyzed by Pearson analysis. (CRC: colorectal cancer, qPCR: Quantitative Real-time PCR)

A
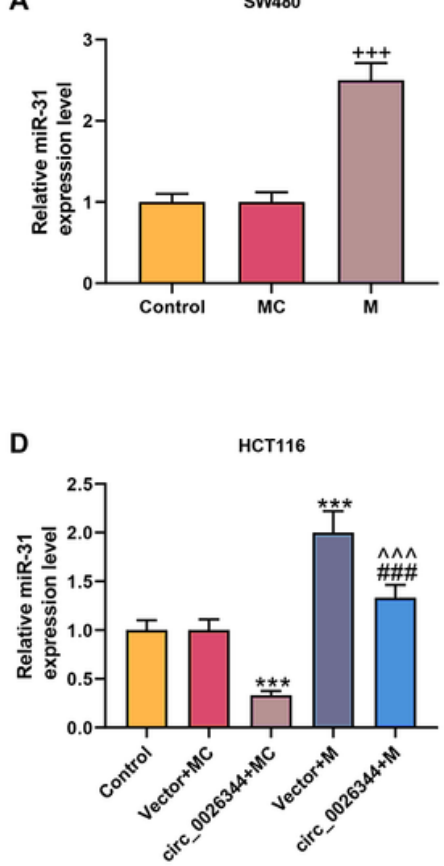

B

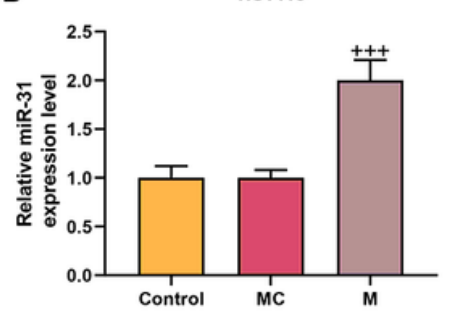

E

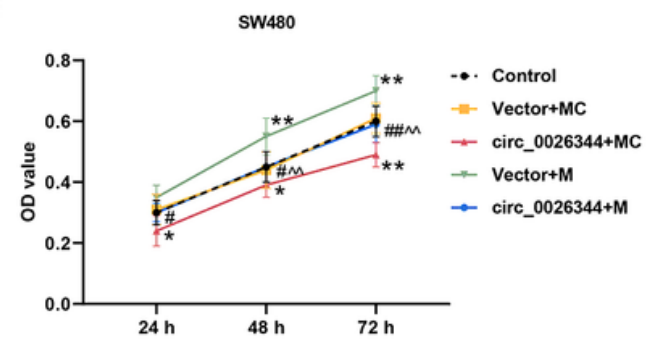

C

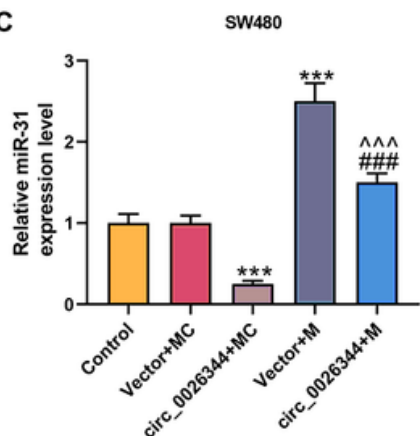

$\mathbf{F}$

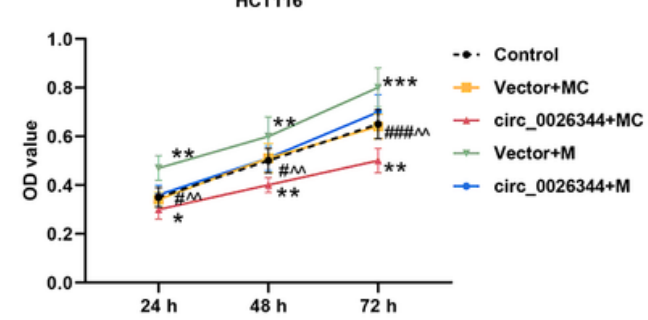

Figure 7

MiR-31 overexpression counteracted the effect of circ_0026344 on the cell viability and the expression of miR-31 in SW480 and HCT116 cells (A-B) The transfection efficiency of miR-31 mimic in SW480 (A) and HCT116 (B) cells were evaluated by qPCR, U6 was used as an internal control. (C-D) The expression of miR-31 in SW480 (C) and HCT116 (D) cells after transfection were detected by qPCR, U6 was used as an internal control. (E-F) The cell viability of SW480 (E) and HCT116 (F) cells after transfection were detected by CCK-8 assays. (+++P $<0.005$, vs. $\mathrm{MC}$; ${ }^{\star} \mathrm{P}<0.05$, ${ }^{\star \star P} \mathrm{P}<0.01$, ${ }^{\star \star \star P}<0.001$, vs. Vector+MC; ${ }^{\wedge \wedge} \mathrm{P}<$ 
0.01, ${ }^{\wedge \wedge \wedge} \mathrm{P}<0.001$, vs. circ_0026344+MC; \#P < 0.05, \#\#P < 0.01, \#\#\#P < 0.001, vs. Vector+M). (qPCR: Quantitative Real-time PCR, M: miR-31 mimic, MC: mimic control)

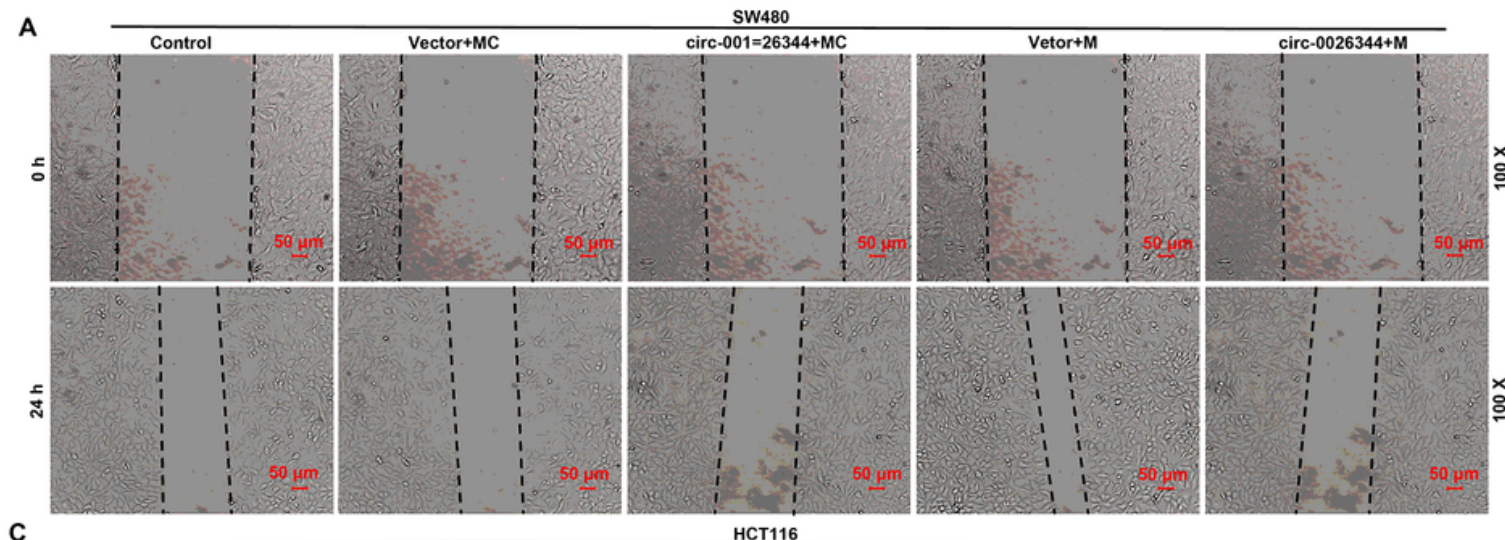

B

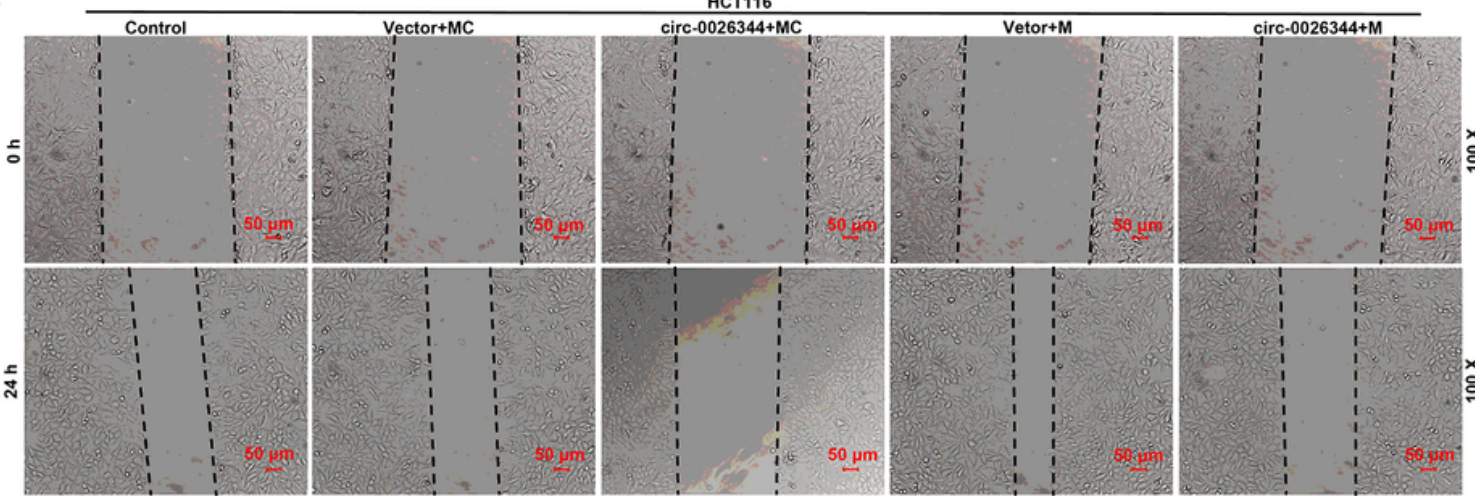

E

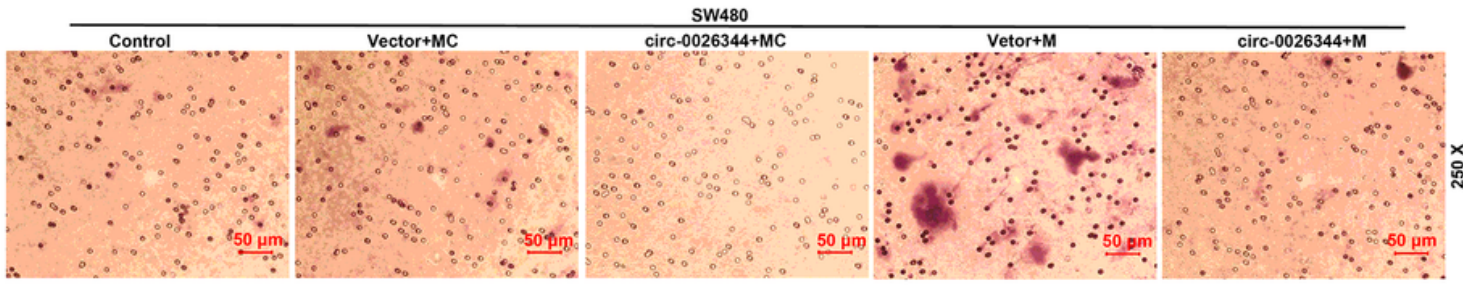

G

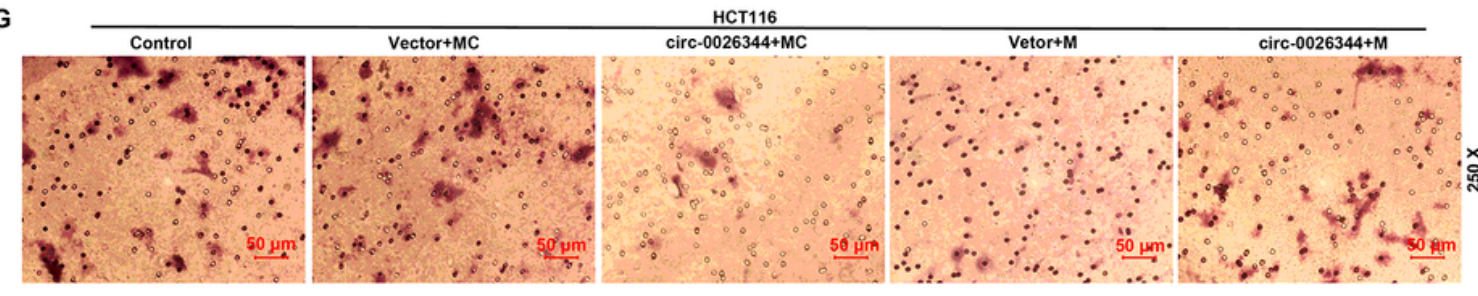

D

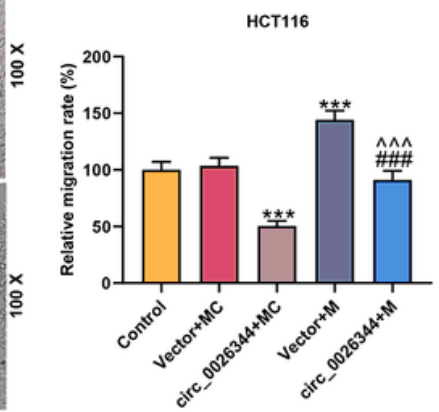

$\mathbf{F}$

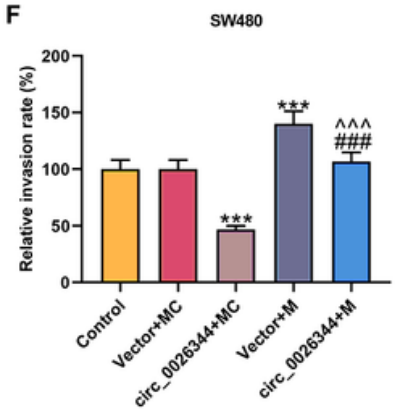

H

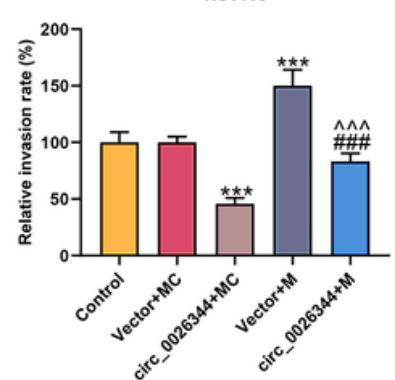

Figure 8

MiR-31 overexpression counteracted the effect of circ_0026344 on the cell migration and invasion of SW480 and HCT116 cells (A-D) The cell migration of SW480 (A-B) and HCT116 (C-D) cells after transfection were detected by wound healing assays. (E-H) The cell invasion of SW480 (E-H) and HCT116 $(\mathrm{G}-\mathrm{H})$ cells after transfection were detected by transwell assays. (***P<0.005, vs. Vector+MC; ${ }^{\wedge \wedge} \mathrm{P}<$ 0.001, vs. circ_0026344+MC; \#\#\#P < 0.001, vs. Vector+M). (M: miR-31 mimic, MC: mimic control) 


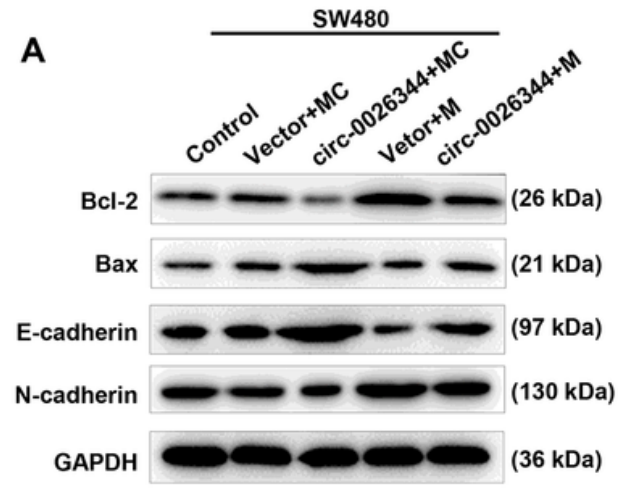

B
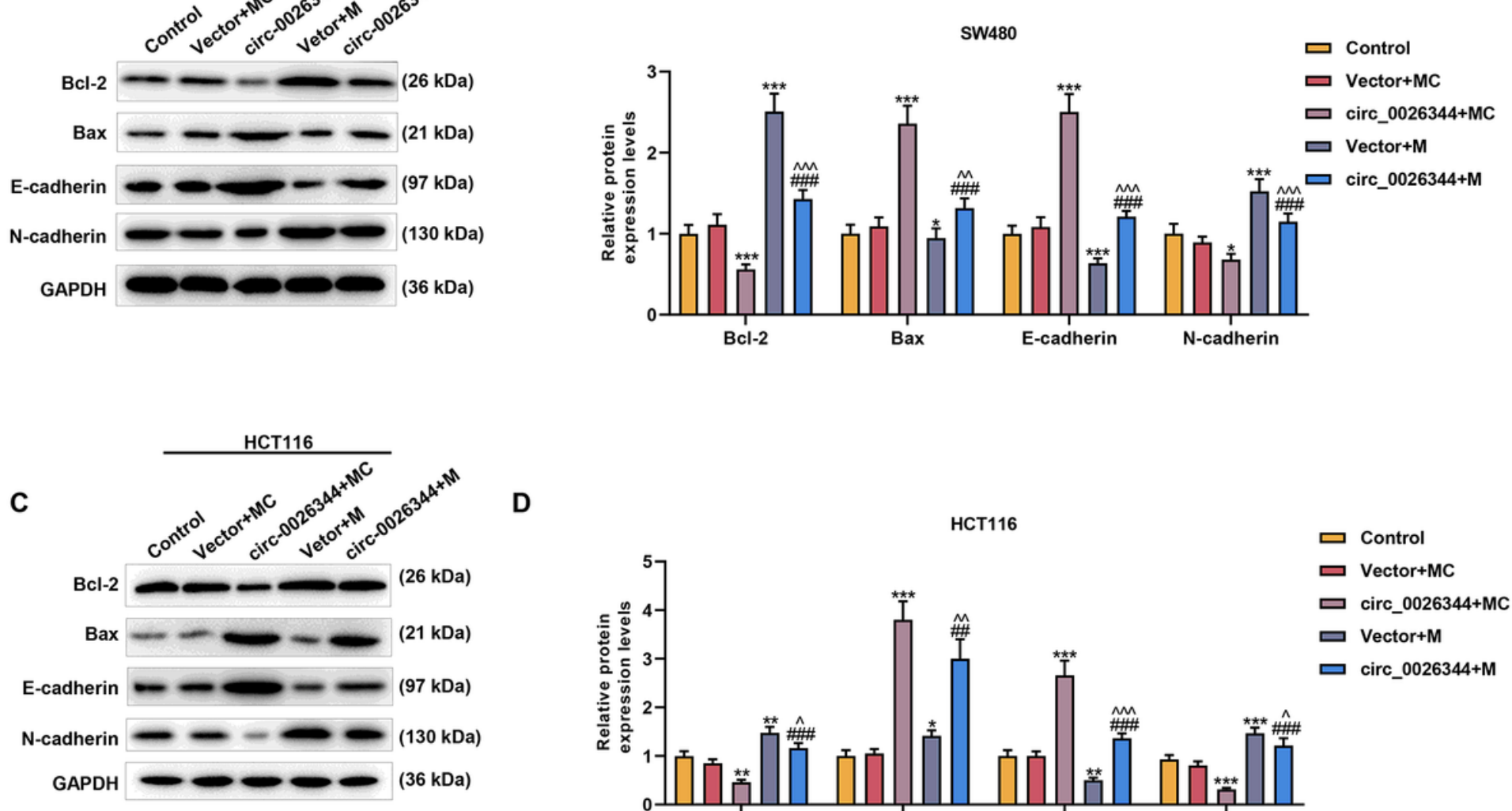

D

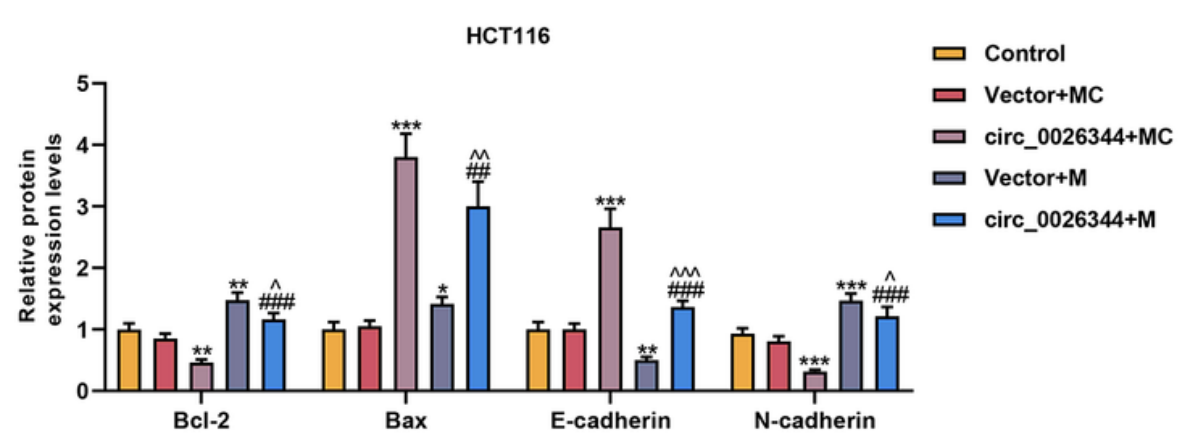

Figure 9

MiR-31 overexpression counteracted the effect of circ_0026344 on the cell migration and invasion of SW480 and HCT116 cells (A-D) The cell migration of SW480 (A-B) and HCT116 (C-D) cells after transfection were detected by wound healing assays. $(\mathrm{E}-\mathrm{H})$ The cell invasion of SW480 (E-H) and HCT116 $(\mathrm{G}-\mathrm{H})$ cells after transfection were detected by transwell assays. ( ${ }^{\star \star \star} \mathrm{P}<0.005$, vs. Vector+MC; ${ }^{\wedge \wedge \wedge} \mathrm{P}<$ 0.001 , vs. circ_0026344+MC; \#\#\#P < 0.001, vs. Vector+M). (M: miR-31 mimic, MC: mimic control) 